\title{
Priming relations in ambiguous noun-noun combinations
}

\author{
CHRISTINA L. GAGNÉ \\ University of Alberta, Edmonton, Alberta, Canada \\ and \\ EDWARD J. SHOBEN \\ University of Illinois at Urbana-Champaign, Champaign, Illinois
}

\begin{abstract}
We conducted two experiments to examine whether the interpretation of an ambiguous noun phrase is influenced by exposure to a similar combination. In Experiment 1, we found that it was easier to verify a definition for a combination (e.g., adolescent doctor, a doctor for adolescents) when the prime used the same relation as the target (e.g., adolescent magazine, a magazine for adolescents; animal doctor, a doctor for animals) than when the prime used a different relation (e.g., country doctor; adolescent experience). In Experiment 2, we found that the interpretation generated for an ambiguous combination was affected by prior exposure to sentences containing a combination with the same modifier or head noun as the target combination. The data are inconsistent with key predictions of schema-based theories of conceptual combination. Although the results do not contradict key assumptions of relationbased theories, modifications to these theories are required to account for these data.
\end{abstract}

As people learn about the world, they create conceptual representations of the categories they encounter. These conceptual representations can be used to interpret (or even produce) new concepts. For example, people can combine the concepts tin and bottle to form the combined concept tin bottle. This process of combining two or more concepts to create a combined concept is called conceptual combination. During conceptual combination, the head noun concept (e.g., bottle) is modified in some way by the modifier concept (e.g., tin). The modifier often specifies the way in which the head noun differs from other members of its category (Clark, 1987; Gelman \& Markman, 1985). For example, a tin bottle differs from most bottles in that it is made of tin, rather than of plastic or glass.

Theories of conceptual combination primarily have taken two approaches: a schema-based approach and a relationbased approach. According to schema-based theories (e.g., Murphy, 1988, 1990; Wisniewski, 1996), a specific dimension within the head noun's representation is selected, and its value is changed to match the value present in the modifier. For example, the value in the FUEL dimension is changed to wood for the combination wood stove. Conse-

This research was supported in part by a fellowship from the Natural Sciences and Engineering Research Council (NSERC) of Canada and by NSERC Research Grant 203054-98 RGPIN to the first author. The first author is grateful to Gina Sohn for her help in data collection for Experiment 1. Correspondence should be addressed to C. L. Gagné, Department of Psychology, University of Alberta, P-217 Biological Sciences Building, Edmonton, AB, T6G 2E9 Canada (e-mail: christina. gagne@ualberta.ca). quently, these theories predict that interpreting a combined concept should take longer if the required dimension is not present in the head noun schema. If relations are represented as dimensions within the head noun concept (as proposed by Wisniewski, 1996), then the frequency with which a relation is used with a given head noun should play a large role in conceptual combination.

In contrast, the competition among relations in nominals (CARIN) theory emphasizes the role of the modifier in relation selection (Gagné, 2001; Gagné \& Shoben, 1997) and draws on linguistic approaches to the interpretation of modifier-noun phrases (Downing, 1977; Levi, 1978). According to this theory, conceptual combination involves selecting a relation that specifies the connection between the modifier and head noun. For example, the relation head noun uses modifier is used to interpret wood stove as "a stove that uses wood." The relation can be used to infer more specific properties of the concept (see Gagné, 2000, in press). According to the CARIN theory, knowledge about the modifier's past usage with various relations is called the modifier's relational distribution, and this distribution is used to determine which relations should be considered during the conceptual combination process. Relations compete with one another such that more strongly activated relations are more likely to be selected than are less activated relations. Once a relation is selected on the basis of the modifier's relational distribution, the head noun is used to determine whether that relation is plausible. In short, the CARIN theory suggests that the head noun plays a role in evaluating the plausibility of the relations suggested by the modifier, but it does not play a role in the initial activation of those relations. 
Recent evidence in the conceptual combination literature appears to favor the relation-based approach. Gagné and Shoben (1997) found that a combination was more readily interpreted when it used a relation that was frequently used with the modifier than when it used a relation that was not often used with the modifier. There was no evidence that the frequency with which the relation was used with the head noun influenced the ease of interpreting a combination. Gagné (2001) and Shoben and Gagné (1997) reported further evidence that the ease of selecting a relation is based on the availability of that relation for the modifier rather than for the head noun. For example, in Gagné (2001), combinations (e.g., student vote) were preceded by a combination sharing either the same modifier (e.g., student accusation) or the same head noun (e.g., employee vote). In addition, the prime combination used either the same relation or a different relation as the target. Although both modifier and head noun primes produced lexical priming, only the modifier prime yielded relation priming. Head noun primes that used the same relation as the target did not yield any more priming than did head noun primes that used a different relation. In contrast, modifier primes with the same relation as the target produced more priming than did modifier primes using a different relation. In sum, research presented by Gagné (2001) and Gagné and Shoben (1997) demonstrates that the ease of selecting a relation depends on how available that relation is for the modifier concept and is not affected by the availability of the relation for the head noun concept. Both findings are consistent with the CARIN theory.

A central tenet of the schema-based approach is that the availability of dimensions within the head noun's schema plays a large role in conceptual combination. Consequently, the findings of Gagné (2001) and Gagné and Shoben (1997) that contradict this claim indicate that the role of the head noun during relation selection should be reconsidered. It is possible that the influence of the head noun is reflected not in its relational distribution (as is the case for the modifier) but in its most recent usage and that this influence is evident only when there is more than one plausible interpretation for a combination. Earlier studies used items for which there was a single dominant interpretation (Gagné, 2001; Gagné \& Shoben, 1997); that is, there was little ambiguity about which relation was most appropriate. If a combination has multiple interpretations (e.g., cat rash), it seems likely that additional information might be required to resolve the ambiguity. If the head noun has been recently used in a combination, then the selection of a relation during the conceptual combination might be influenced by this recent usage. Thus, for example, if one had recently encountered rash in the context of heat rash, one might be more likely to interpret cat rash as "a rash caused by a cat." On the other hand, if one had encountered rash in the context of leg rash, one might interpret cat rash as "a rash on a cat." Our aim was to determine whether this scenario is correct, and the experiments provided an additional test of schema-based and relation-based theories of conceptual combination.

\section{Overview of the Experiments}

In the present experiments, we used combinations for which there were at least two nearly equally plausible interpretations. We used ambiguous combinations because it is likely that this situation might require the head noun to play a greater role in the selection of the relation than was observed in previous studies. Our aim was to determine whether a concept's recent usage in a combined concept affects the comprehension of a subsequent combination containing the same concept. Recall that the schemabased (Murphy, 1988, 1990; Wisniewski, 1996) and relationbased (Gagné \& Shoben, 1997) theories emphasize different constituents. It therefore is probative to examine the effect of both constituents. In Experiment 1, we examined the time required to verify a particular definition for a target combination following various prime combinations. The prime combinations used either the same head noun or the same modifier as the target combination. In addition, the prime's meaning was based on the target's dominant relation, was based on the target's subdominant relation, or was unrelated to the target's two most common meanings. To ensure that the results obtained in Experiment 1 were not specific to the verification task and to generalize our findings to a nonspeeded task, we conducted an experiment (Experiment 2) that used an off-line task in which the participants read a series of sentences containing the prime combinations and then (after a delay) generated their own interpretations for the ambiguous target combinations.

\section{EXPERIMENT 1}

The goal of Experiment 1 was to determine whether repetition of either a modifier or a head noun can influence subsequent comprehension of an ambiguous combination. Gagné and Shoben (1997) demonstrated that head nouns and modifiers vary in terms of how much prior usage with various relations affected the ease of comprehending novel combinations. The use of a common relation rather than a rare relation for the modifier led to easier comprehension of the combination. In contrast, there was no effect of frequency of relation for the head nouns on comprehension. However, these findings do not necessarily imply that the head noun never plays a role in the selection of a relation. Instead, it might be that distributional information about the head noun relations is not as readily available as is information about how the modifier tends to be used. If so, it might still be possible for the head noun to influence the interpretation of a combined concept if there is a recent example of a combination containing the same head noun available. That is, rather than being influenced by how the head noun is used in general, the comprehension of combinations might be affected by how the head noun was most recently used. The presence of a recent prime might make relational information about the head noun more available, especially when there is ambiguity about which relation should be selected. In this experiment, we examined whether a recent example of a 
combination containing the same head noun influences the interpretation of a combination.

For the target combinations, we selected combined concepts that were ambiguous in that they could have at least two meanings (as judged by the results of a norming study in which participants provided definitions for various combinations). For example, adolescent doctor could mean either "a doctor who is an adolescent" or "a doctor for adolescents." To determine whether the interpretation of this combination is influenced by a recent example of a similar combination, we constructed prime combinations that used the target's dominant relation, the target's subdominant relation, or a relation other than the target's two most frequent relations. In addition to varying the type of relation, we also varied whether the prime and the target used the same modifier or the same head noun.

If the head noun plays an important role in selecting the appropriate relation, as predicted by schema-based theories, then it should be easier to interpret adolescent doctor as "a doctor who is an adolescent" when the phrase male doctor has been recently seen than when the phrase animal doctor has been seen. The opposite should be true if adolescent doctor is to be interpreted as "a doctor for adolescents." However, if the head noun does not play a role in the selection of a relation during conceptual combination, then it should not matter which combination precedes the target combination. Instead, the meaning that is the more dominant (or more common) should be the easiest to derive.

As for a recent example of a combination containing the same modifier as the target, previous research suggests that a combination with a similar modifier should influence response time (RT; Gagné, 2001; Gagné \& Shoben, 1997). As previously mentioned, Gagné and Shoben (1997) have shown that conceptual combination is guided by information about how the modifier tends to be used. In the CARIN model, this information is referred to as the modifier's relational distribution. This relational distribution is based on people's experience with combined concepts containing that modifier. Thus, one extension of the CARIN model might lead one to expect a prime combination to alter the interpretation of a later combination sharing the same modifier. This influence would occur if the prime combination makes a particular relation more accessible in the modifier's relational distribution. That is, a recent example of the modifier being used with a particular relation may make that relation more accessible in the relational distribution than it might be otherwise. However, it is not entirely obvious that the interpretation of a combined concept will necessarily be influenced by prior exposure to another combined concept with the same modifier. For the prime combination to strongly influence the interpretation of a combination, the prime must make the relation more accessible than the most available relations in the modifier's relational distribution, because the relation that was used in the prime combination must compete successfully against the most highly available relations in the modifier's relational distribution before it can be selected during the interpretation of the target combination. Given that the modifier's relational distribution is based on people's lifelong experience with combined concepts containing that modifier, it might very well be that a single exposure of a particular relation will have little effect on this relational distribution.

\section{Method}

Norming study. The authors generated 112 combinations that had at least two interpretations. For example, cloth money could refer to either "money that is made of cloth" or "money for cloth." Five randomized lists of all 112 items were created, and one list was presented to each of 37 participants at the University of Illinois. These participants provided a definition for each combination. The first author tabulated the frequency that each definition was used for a particular combination. The definition that was used most often for a combination was deemed the dominant meaning, and the second most frequent definition was deemed the subdominant meaning.

Materials. Thirty-six ambiguous combinations were selected from the items in the norming study. These combinations were used as target combinations. For each target, we created prime combinations that varied along two dimensions. First, the prime used either the same modifier or the same head noun as the target combination. Second, the prime could be interpreted using the same relation as the target's dominant meaning, the target's subdominant meaning, or a relation that was unrelated to either the dominant or subdominant relation of the target. These two factors were crossed to produce six prime conditions: modifier repeated-subdominant relation (MS), modifier repeated-dominant relation (MD), modifier repeated-unrelated relation (MU), head repeated-subdominant relation (HS), head repeated-dominant relation (HD), and head repeated-unrelated relation (HU). Examples of the six prime types that were used for adolescent doctor are given in Table 1 (see the Appendix for a complete list of experimental items). The subdominant meaning for this target item was "a doctor who is an adolescent," and the dominant meaning was "a doctor for adolescents." Although the prime items were based on either the subdominant meaning or the dominant meaning of the target, the target sentence always required the participants to verify a combination's subdominant meaning. For the present example, the target sentence was "adolescent doctor $=$ a doctor who is an adolescent."

One hundred eight filler pairs were also created in which the prime, the target, or both items were shown with an incorrect definition. For

Table 1

Example Stimuli for Experiment 1

\begin{tabular}{llll}
\hline & \multicolumn{3}{c}{ Prime's Relation } \\
\cline { 2 - 4 } Repeated Constituent & \multicolumn{1}{c}{ Subdominant } & \multicolumn{1}{c}{ Dominant } & \multicolumn{1}{c}{ Unrelated } \\
\hline Modifier & $\begin{array}{l}\text { adolescent student } \\
\text { male doctor }\end{array}$ & $\begin{array}{l}\text { adolescent magazine } \\
\text { animal doctor }\end{array}$ & $\begin{array}{l}\text { adolescent experience } \\
\text { country doctor }\end{array}$ \\
\hline
\end{tabular}

Note-The target combination (adolescent doctor) was always displayed with the subdominant meaning ("a doctor who is an adolescent"). 
36 filler pairs, the prime was shown with a correct definition, and the target had an incorrect definition. For 36 filler pairs, the prime had an incorrect definition, and the target had a correct definition. For the remaining 36 filler pairs, both the prime and the target were shown with an incorrect definition. In addition, the prime and the target shared the same modifier for half of the filler pairs. For the other half of the filler pairs, the prime and the target shared the same head noun.

Procedure. Each participant saw the entire set of target combinations, and each target item was seen only once. Six stimulus lists were created, such that there was an equal number of each prime type on each list. Across all lists, each target was seen with each prime combination. The 36 experimental pairs of prime and target items were presented in a randomized order along with 108 filler pairs. The prime combination was presented first. Then, following the participant's response, the target combination was presented. There was nothing in the method of presentation to indicate that the prime and the target were connected. The way in which the prime and target combinations were displayed was identical. Each trial began with the question "Ready?" on the computer screen, and the participants pressed the space bar when they wanted to see the next item. After the space bar was pressed, the next combination along with a possible definition (e.g., "adolescent doctor $=$ a doctor who is an adolescent") was displayed in the center of the computer screen. The participants pressed either the " $J$ " or the " $F$ " key on the keyboard to indicate whether the definition was acceptable. The keys were labeled such that the key marked "Acceptable" corresponded to the participant's dominant hand, and the other key was labeled "Unacceptable."

Participants. Seventy-two undergraduates from the University of Western Ontario participated in the study for partial course credit. All were native speakers of English.

\section{Results and Discussion}

The data for the target items are of primary interest and are shown in Table 2. Recall that the target was always seen with the subdominant relation. There was a main effect of prime relation $\left[F_{1}(2,142)=10.73, p<.0001 ; F_{2}(2,70)=\right.$ $4.70, p<.01]$. RTs to the target were $224 \mathrm{msec}$ faster when preceded by the subdominant prime than when preceded by the dominant prime $\left[F_{1}(1,142)=20.90, p<.0001\right.$; $\left.F_{2}(1,70)=8.79, p<.01\right]$. In addition, RTs to the target were $143 \mathrm{msec}$ faster when preceded by the subdominant prime than when preceded by the unrelated prime $\left[F_{1}(1,142)=8.62, p<01 ; F_{2}(1,70)=4.64, p<.05\right]$. Thus, the participants were faster to respond to the target when the prime's definition used the same relation as the target than when the prime's definition used a different relation. These results indicate that a recently viewed combination can influence the ease of selecting a relation for a subsequent combination such that it is easier to interpret

Table 2

Mean Response Times (RTs), Standard Deviations (SDs), and Accuracy Rates for Target Items in Experiment 1 Prime

\begin{tabular}{clcccc}
\hline \multicolumn{2}{c}{ Repeated Constituent } & Relation & RT (msec) & SD & Accuracy \% \\
\hline Modifier & Subdominant & 2,103 & 457 & 85 \\
Modifier & Dominant & 2,288 & 579 & 74 \\
Modifier & Unrelated & 2,232 & 519 & 78 \\
Head noun & Subdominant & 2,068 & 555 & 89 \\
Head noun & Dominant & 2,331 & 626 & 66 \\
Head noun & Unrelated & 2,227 & 649 & 78 \\
\hline
\end{tabular}

the subsequent combination when it requires the same relation as the previous combination.

Most importantly, the analysis revealed that the influence exerted by the modifier and head noun primes did not differ. Overall, the mean in the head noun condition $(2,209 \mathrm{msec})$ was nearly identical to the mean in the modifier condition $(2,208 \mathrm{msec})$. There was no main effect of which constituent was repeated, and there was no interaction between prime relation and repeated constituent (all $\left.F_{\mathrm{s}}<1\right)$. Importantly, the facilitation observed from the subdominant prime was observed for both the modifier prime conditions and the head noun prime conditions. The difference between the MS and MU conditions was $129 \mathrm{msec}$ and was significant in the subject analysis $\left[F_{1}(1,142)=3.89, p<.05\right]$ but did not reach conventional significance levels in the item analysis $\left[F_{2}(1,70)=\right.$ $2.08, p>.10]$. Similarly, the $159-$ msec difference between the HS and HU conditions was reliable in the subject analysis $\left[F_{1}(1,142)=5.83, p<.05 ; F_{2}(1,70)=2.98\right.$, $p>.05]$.

The dominant relation primes did not produce interference. The 56-msec difference between the MD and MU conditions was not significant $\left(F_{\mathrm{S}}<1\right)$, nor was the 104msec difference between the HD and HU conditions $\left[F_{1}(1,142)=2.52, p>.10 ; F_{2}(1,70)<1\right]$. It is unlikely that our failure to detect a difference between these conditions was due to lack of power, because a power calculation using GPOWER (Erdfelder, Faul, \& Buchner, 1996) indicated that power was .99 , based on an alpha of .05 and an $f$ of .25 (which corresponds to a medium effect size; see Cohen, 1988).

The accuracy data also indicate that the prime combination influenced the interpretation of the target combination. Again, there was a main effect of prime relation $\left[F_{1}(2,142)=40.29, p<.0001 ; F_{2}(2,70)=26.75, p<\right.$ $.0001]$. Accuracy rates in the subdominant condition were higher than in the dominant condition $\left[F_{1}(1,142)=19.52\right.$, $\left.p<.0001 ; F_{2}(1,70)=53.50, p<.0001\right]$. In addition, accuracy rates were higher in the subdominant condition than in the unrelated condition $\left[F_{1}(1,142)=20.77, p<\right.$ $\left..0001 ; F_{2}(1,70)=13.91, p<.001\right]$. Thus, the accuracy data parallel the RT data in that performance was improved when the prime and the target shared the same relation relative to when the prime and the target used different relations.

Consistent with what was observed in the RT data, there was no main effect of which constituent was repeated $\left(F_{\mathrm{S}}<1\right)$. Importantly, both the modifier and the head noun primes yielded facilitation. The difference between the MS and MU conditions was significant $\left[F_{1}(1,142)=4.84\right.$, $\left.p<.05 ; F_{2}(1,70)=4.49, p<.05\right]$, as was the difference between the HS and HU conditions $\left[F_{1}(1,142)=14.00\right.$, $\left.p<.05 ; F_{2}(1,70)=14.58, p<.05\right]$.

Unlike the response time data, the accuracy data indicate that the dominant prime made the target more difficult to interpret; the difference between the dominant and unrelated conditions was significant $\left[F_{1}(1,142)=19.52\right.$, 
$\left.p<.0001 ; F_{2}(1,70)=12.85, p<.0001\right]$, and there was a significant interaction between the repeated constituent and prime relation factors $\left[F_{1}(2,142)=4.31, p<.05\right.$; $\left.F_{2}(2,70)=4.21, p<.05\right]$. Interference from the dominant prime was observed when the head noun was in common $\left[F_{1}(1,142)=17.35, p<.0001 ; F_{2}(1,70)=16.37, p<\right.$ $.0001]$, but not when the modifier was in common $\left[F_{1}(1,142)=2.55, p>.10 ; F_{2}(1,70)=2.75, p>.10\right]$. Thus, there is some indication in the accuracy data that the modifier and the head noun primes might yield somewhat different effects, in that interference was obtained only from the head noun prime.

Taken together, the data indicate that the conceptual combination process can make use of information about how the head noun and the modifier were most recently used, even though it does not appear to make use of how the head noun is used in general (as suggested by Gagné $\&$ Shoben, 1997). Apparently, information about how the head noun has been used is not readily available unless there is a very recent example of another combination containing that same head noun. In addition, it appears that the influence of the head noun prime occurs only when the target combination is ambiguous: Gagné (2001) used unambiguous target combinations and failed to find priming when the prime and target combination shared the same head noun.

A second implication is that a modifier's relational distribution can be altered by prior exposure to a combination containing the same modifier. In other words, general information about the relations with which the modifier tends to be used is not the modifier's only source of influence on relation selection. Instead, a modifier's recent usage can increase the availability of a particular relation such that it can be selected more readily if it is required to interpret a subsequent combination.

\section{EXPERIMENT 2}

In Experiment 1, we used an on-line verification task to investigate whether the interpretation of a combined concept is influenced by the presence of a related combination. In Experiment 2, we examined the influence of a related prime in a situation in which the participant must generate (rather than verify) a definition for an ambiguous combination. The participants read a group of sentences (e.g., "The tiny puppy was cold, so his owner made a dog sweater for him"), each of which contained a prime combination (e.g., dog sweater). Subsequently, the participants provided definitions for the corresponding target combinations (e.g., goat sweater).

Using an interpretation task enabled us to determine whether the results obtained with the verification task generalize to a nonspeeded task. If so, the data from the present experiment would allow us to broaden our conclusions and show that the influence of a recent modifier or a head noun prime is not confined to a situation in which the prime combination is used with a definitional statement.

On the basis of the previous results, one would expect the prime combination to bias people's interpretations of an ambiguous combination. When defining an ambiguous combination, there should be a tendency for people to provide an interpretation that uses the same relation as the one embodied in the previous prime combination. For example, if people are influenced by the recent modifier prime, they should be more likely to define goat sweater as "a sweater for a goat" if they had previously seen a sentence containing goat collar ("a collar for a goat") than if they had seen a sentence containing goat rug ("a rug made of goat"). Likewise, if people are influenced by the recent head noun prime, they should be more likely to define goat sweater as "a sweater for a goat" if they had previously seen a sentence containing dog sweater ("a sweater for a dog") than if they had seen a sentence containing rayon sweater ("a sweater made of rayon").

\section{Method}

Materials. Thirty-six ambiguous combinations were selected from the items used in the norming study (see the Method section of Experiment 1 for a description of the norming study). These combinations were used as target combinations. For each target, we created prime combinations that varied along two dimensions. First, the prime used either the same modifier or the same head noun as the target combination. Second, the prime could be interpreted using the same relation as the target's dominant meaning or the target's subdominant meaning. Crossing these two variables produced four experimental conditions: modifier repeated-subdominant relation (MS), modifier repeated-dominant relation (MD), head repeated-subdominant relation (HS), and head repeated-dominant relation (HD). We created a sentence for each prime combination. The sentences implied the meaning of the prime combination but did not explicitly state it. For example, animal doctor (one of the primes for adolescent doctor) was used in the following sentence: "James Herriot, author of several books, is a famous animal doctor."

Procedure. The materials were placed into four booklets, such that, across the booklets, each target item appeared with each prime type. Each target combination was presented only once per booklet, and each participant received one booklet. Within each booklet, the items were randomized and divided into four blocks. First, the participants read nine study sentences. These study sentences were the prime sentences described above. All nine sentences were printed on a single sheet of paper, and the participants were given several minutes to read the sentences. The next page contained a list of 18 words: 9 words from the study sentences and 9 new words. None of the words was from the prime combinations. The participants were instructed to circle the words that had appeared on the previous page. This memory test was done to ensure that the participants were reading the study sentences and to provide a distractor task between the presentation of the prime and target combinations. Finally, the next page listed the nine target combinations that corresponded to the prime combinations used in the study sentence for that block. For example, if the sentence "James Herriot, author of several books, is a famous animal doctor" appeared among the study sentences, then the target combination adolescent doctor was presented after the distractor task. The participants provided a definition for each item on the line next to the combination. This sequence was repeated for each block of items, until all 36 target combinations had been presented.

Participants. Forty undergraduate students from the University of Illinois participated in the study for partial course credit. They were all native speakers of English.

\section{Results and Discussion}

The interpretations were classified into one of three categories: dominant, subdominant, and other. The dominant 
and subdominant classifications were based on the results of the norming study reported in the Method section of Experiment 1 . Overall, $90 \%$ of the interpretations used either the subdominant or the dominant interpretation. The other category consisted of responses that used an interpretation other than the subdominant or the dominant interpretation or did not clearly define the combination. For example, "a basket of fruit" for fruit wrap and "a pretty lamp" for picture lamp were both classified as other because the intended meaning was not clear. In the data analysis, the other category was not used.

Table 3 shows the proportion of dominant and subdominant interpretations in each condition. A key issue is whether the presence of the prime combination changes the proportion of dominant and subdominant interpretations. To answer this question, we used a normal approximation to the binomial to determine whether the number of subdominantinterpretationscorresponded to what would be expected on the basis of the number of subdominant interpretations that were observed in the norming study reported in the Method section of Experiment 1 (in which the combinations were not preceded by prime combinations). On the basis of the norming study, the predicted proportion of dominant interpretations was .62, and the proportion of subdominant relations was .38 (when the other category was not included in the analysis). Note that a comparison using the number of dominant interpretations would yield the identical absolute $z$-value as a comparison using the number of subdominant interpretations; so the choice of whether to use the number of subdominant or dominant interpretations is arbitrary.

The influence of the dominant prime on the number of subdominant interpretations was not statistically significant. When the prime used the target's dominant interpretation and the same modifier as the target, the proportion of subdominantinterpretations did not differ significantly from what was obtained in the norming study $(z=0.77$, $p>.10)$. The same was true when the prime used the same head noun as the target and the target's dominant interpretation $(z=1.22, p>.10)$. These results suggest that primes using the same relation as the target combination's dominant interpretation did not greatly alter the proportion of subdominant and dominant interpretations given to the target combinations.

In contrast, the primes using the target's subdominant interpretation did have a significant influence on the proportion of dominant and subdominant interpretations. This finding held for both the modifier prime condition and the head noun prime condition. In the modifier prime condition, the number of subdominant interpretations was significantly higher than what would be predicted by the norming study $(z=3.40, p<.0001)$. In the head noun prime condition, the number of subdominant interpretations was significantly higher than predicted $(z=4.76$, $p<.0001)$. These results show a clear effect of both the modifier prime and the head noun prime. Prior exposure to a combination that used the same relation as the target's subdominant interpretation increased the number of subdominant interpretations generated for the ambiguous combination.

Overall, the results of Experiment 2 are congruent with the results of Experiment 1 in that they demonstrate that both the modifier prime and the head noun prime influence the interpretation of an ambiguous combination. Thus, the results of Experiment 2 indicate that the findings obtained in Experiment 1 are not specific to a speeded task but instead generalize to a situation in which the participants generate their own interpretations.

One difference between Experiment 1 and Experiment 2 is that there was no influence of the dominant prime in Experiment 2. It appears that the dominant prime affects the ease of selecting a relation (as indicated in Experiment 1) but does not necessarily affect the propensity to use the dominant relation. Prior exposure to a prime using the dominant meaning of the target combination did not increase the relative number of dominant interpretations generated for the ambiguous target combination, even though the dominant primes did have an influence in the on-line task (as indicated by a lower accuracy rate following the head noun prime). Perhaps the number of dominant interpretations generated by the participants was already at ceiling, so that the dominant prime was unable to significantly increase the number of dominant interpretations provided. Recall that, in the norming study, $62 \%$ of interpretations for the ambiguous target combinations used the dominant relation. Although this percentage is not a statistical ceiling, it is worth noting that the percentage of interpretations with the dominant interpretation might be near a theoretical ceiling if the selection of the dominant relation is constrained by the availability of other plausible relations and by other linguistic and conceptual factors (e.g., affordances; Kaschak \& Glenberg, 2000). Put another way, the use of the dominant relation when the target combination is presented in isolation might already reflect the highest

Table 3

Proportion of Dominant and Subdominant

Interpretations in Experiment 2

\begin{tabular}{cccccc}
\hline \multicolumn{2}{c}{ Prime } & & & \multicolumn{2}{c}{ Interpretation Given to Target } \\
\cline { 5 - 6 } \cline { 5 - 5 } Repeated Constituent & Prime's Relation & & Dominant & Subdominant \\
\hline Modifier & Dominant & & .60 & .40 \\
Modifier & Subdominant & & .53 & .47 \\
Head noun & Dominant & & .58 & .42 \\
Head noun & Subdominant & & .49 & .51 \\
\hline
\end{tabular}


proportion of responses given to any relation for an ambiguous combination. If so, it might be very difficult to increase the use of this relation. Another possibility is that prior exposure to the subdominant relation might have increased the strength of this relation relative to other nondominantrelations, and this might have allowed the subdominant relation to compete more successfully with the dominant relation. In contrast, prior exposure to the a combination using the target's dominant relation might not have greatly increased the availability of the dominant relation because the strength of the dominant relation was already higher than all other relations and any additional increase in strength would not provide much advantage over the other relations (relative to the situation in which the dominant relation was not primed). Consequently, exposure to the dominant relation prime might not have increased the use of the dominant relation during the interpretation of the target, even though the time required to use the dominant relation was reduced (as observed in Experiment 1).

\section{GENERAL DISCUSSION}

The results of Experiments 1 and 2 demonstrate that the interpretation of an ambiguous combined concept can be influenced by recent exposure to a combined concept containing the same modifier or the same head noun. Although the psychologicalliterature contains many demonstrations that prime items can influence responses to subsequent target items (see Meyer \& Schvaneveldt, 1971; Meyer, Schvaneveldt, \& Ruddy, 1974; Neely, 1977; Onifer \& Swinney, 1981), much of this literature has focused on lexical priming with single words, and there have been few demonstrations that conceptual combination can be affected by prior exposure to related combinations. Within the conceptual combination literature, there has been some indication that prior discourse context influences the interpretation of a combined concept (Gagné \& Murphy, 1996; Gerrig \& Bortfeld, 1999; Gerrig \& Murphy, 1992). However, our results demonstrate that a preceding discourse context is not required to influence the interpretation of a combined concept. Instead, we show that a single combination can affect the interpretation of a subsequent combination. This finding suggests that the priming context need not be rich or complex for priming to occur.

Another way that our results differ from the usual priming effect is that the results of our off-line task demonstrate that the influence of the prime is evident not only in a verification task but also in a task in which the participants generate their own interpretations. These data suggest that the priming observed in the on-line task is not restricted to lexical priming. Instead, the ability to select a particular relation is affected by prior exposure to a combination containing a similar relation.

Our data have several implications for the viability of both the schema-based (e.g., Murphy, 1988, 1990; Smith \& Osherson, 1984; Smith, Osherson, Rips, \& Keane, 1988; Wisniewski, 1996) and the relation-based (e.g. Gagné,
2001; Gagné \& Shoben, 1997) approaches. Before considering these implications, we will first outline the differences between the CARIN theory (Gagné \& Shoben, 1997) and the schema modification theory (Murphy, 1988, 1990) that are relevant for this set of experiments. The relationlinking process in Wisniewski's (1996) dual-process theory shares the same key assumptions as the schema modification theory, so the discussion of the schema modification theory also applies to the dual-process theory. The CARIN theory and the schema modification theory differ in two ways. The first difference between the two models is the role that the modifier and the head noun play during conceptual combination. The schema modification theory asserts that properties of the head noun play a large role in conceptual combination. The ease with which a combination can be interpreted is influenced by the existence of a particular dimension within the head noun. If the required dimension does not exist, then the combination will be judged as nonsensical unless the dimension can be created. The modifier's role during conceptual combination is to fill a particular dimension within the head noun. In this respect, the influence of the modifier is tied largely to the head noun. For the CARIN theory, however, the modifier is much less dependent because it plays a strong role in the selection of a relation. The frequency with which particular relations have been used with a given modifier strongly influences the ease with which a combination can be interpreted (Gagné, 2001; Gagné \& Shoben, 1997).

A second difference concerns the specificity of the modifications that are made during the interpretation of a combined concept. For the schema modification theory, conceptual combination involves altering a specific dimension within the head noun's representation. For the CARIN theory, however, conceptual combination is primarily based on a general relation. To illustrate this difference, consider the combination wood stove. According to the schema modification theory, a specific dimension (e.g., FUEL) is selected. The same dimension is selected for combinations such as diesel truck and gas lamp because the modifiers all represent types of fuel. According to the CARIN theory, an important component of conceptual combination is the selection of a general relation (e.g., noun uses modifier). The same relation can be used for other combinations, such as honey remedy, steam brake, or smoke signals, even though the modifiers in these examples do not denote types of fuel.

Our data suggest that the relation-based CARIN theory and the schema-based theories are inadequate. The original CARIN theory outlined by Gagné and Shoben (1997) proposes that the more frequently the required relation was used with a particular modifier, the easier it was to interpret the combination. However, our present results suggest that the modifier's relational distribution is not the only source that influences the selection of a relation. A recent similar combination also plays a role. Thus, the CARIN theory must be modified to allow for the use of a recent combination. 
One way to modify the CARIN theory is to propose that the modifier and the head noun each contribute to the selection of a relation in a different way. As proposed in the original CARIN model, the modifier's relational distribution is one source of information that is used during the selection of a relation. The presentation of a combination might alter the relational distribution that is associated with the modifier of that particular combination. That is, the strength of the relation noun for modifier in the relational distribution for adolescent is somewhat increased following the presentation of the combination adolescent magazine. A second source of information that might be consulted during conceptual combination concerns the head noun. The present data suggest that the head noun's contribution to the selection of the relation comes from the most recent combination containing that head noun, rather than from knowledge about the relations with which the head noun tends to be used in general. That is, rather than storing a relational distribution for the head noun, people might store only information about the head noun's most recent usage. In addition, it appears that this information about the head noun's recent usage is evident only when the target combination is ambiguous: Gagné (2001) did not find evidence of relation priming from a head noun prime when the target combination had a single dominant interpretation. However, when a combination has two almost equally plausible interpretations, as was the case in the present experiments, previously viewed examples are used to resolve ambiguity about which interpretation is most appropriate.

Our results also have implications for the viability of schema-based theories. We will concentrate on the schema modification theory and dual-process theory. These theories do not account for our data for a number of reasons. First, the theories use dimensions rather than relations and therefore cannot explain the present set of results. If, for example, the dimension for gender is altered in male doctor, then why does prior exposure to this combination facilitate the comprehension of adolescent doctor where the altered dimension (age) is quite different? Note, however, the CARIN theory can account for why male doctor helps the interpretation of adolescent doctor: Both combinations use the same relation (noun is modifier). This problem is not specific to the predication relation noun is modifier. For example, the combinations Christmas wreath and home wreath both use the same general relation (noun for modifier) but different dimensions. Christmas is a filler for a dimension that specifies the time of year during which the wreath is displayed, but home is a filler for a dimension that specifies the place at which the wreath is designed to be hung. To take another example, balloon gown and curtain gown both use the noun resembles modifier relation. However, balloon alters the dimension that specifies the shape of the skirt of the gown, whereas curtain fills the dimension that specifies the fabric from which the gown is made. Thus, according to the dimension-based theories, Christmas wreath should not have aided the inter- pretation of home wreath, and balloon gown should not have aided the interpretation of curtain gown.

A second problem with schema-based theories is that several aspects of these theories suggest that repeating the modifier should not influence the comprehension of a subsequent combination. First, there is no mechanism whereby the representation of the modifier is altered in any way by the combination process. It is clear from the description of the model that the representation of the head noun is changed as a result of the combination process. That is, existing dimensions within the head noun schema are altered by placing the modifier in the appropriate dimension, or, if necessary, additional dimensions are added to the head noun schema. On the other hand, although the modifier provides the values with which the dimensions in the head noun are filled, the representation of the modifier is apparently unchanged after the combination process. If so, then repeating the modifier should have no effect. Contrary to this prediction, we found that the modifier prime does influence the accuracy with which a combined concept can be interpreted, as well as the ease of verifying a definition for the target (as indicated by the subject analysis in Experiment 1). In addition, a modifier prime using the same relation as the target's subdominant interpretation increases the use of that relation when people are asked to provide an interpretation for the target (as indicated in Experiment 2).

One could add assumptions to the schema modification theory and dual-process theory to allow them to predict that repeating the modifier should influence the interpretation of a combined concept. That is, one could assume that selecting a slot should be easier if that modifier has recently selected the same slot. However, adding this property to these two theories seems inconsistent with the notion that the meaning of a modifier changes depending on the head noun with which it is paired. For example, the concept red is not equivalent in red hair and red apple. Indeed, Murphy (1990) criticizes the notion that a modifier should have the same effect on all nouns (as suggested by Smith \& Osherson, 1984). Given that the meaning of the modifier apparently differs depending on the head noun with which it is combined, repeating the modifier should not benefit conceptual combination because, when the second time a modifier is encountered, it would not be interpreted in the same sense as the initial presentation. In most cases, the meaning of the modifier would not be appropriate for the new head noun. For example, a red apple is not a coppery-orange shade of red (as is red hair).

\section{Conclusions}

We have demonstrated that the selection of a relation for an ambiguous conceptual combination is influenced by recent exposure to constituent concepts. Moreover, this conclusion holds for recent exposure to both head nouns and modifiers; we found no evidence to suggest that the amount of priming was greater for either of these constituents. These results require modification of relation-based the- 
ories to take into account effects of prior experience on the selection of a relation. As currently constituted, the CARIN theory ascribes no role to such experiential effects and, instead, relies exclusively on a relational distribution for the modifier. The most promising revision suggests that prior exposure can influence the distribution of relational information and that a recent example of a combination using the head noun might be retrieved to resolve relation ambiguity. The results pose more serious problems for schema-based accounts, largely because we found priming in situations in which the dimension (a critical component of these accounts) was not the same between prime and target. Moreover, these theories at least imply that the effects for the head noun should be greater than those for the modifier and that no priming should be obtained when only the modifier is in common between the prime and the target.

\section{REFERENCES}

ClARK, E. (1987). The principle of contrast: A constraint on acquisition. In B. MacWhinney (Ed.), Mechanisms of language acquisition (pp. 133). Hillsdale, NJ: Erlbaum.

CoHen, J. (1988). Statistical power analysis for the behavioral sciences. Hillsdale, NJ: Erlbaum.

Downing, P. (1977). On the creation and use of English compound nouns. Language, 53, 810-842.

ERdfelder, E., FAul, F., \& Buchner, A. (1996). GPOWER: A general power analysis program. Behavior Research Methods, Instruments, \& Computers, 28, 1-11.

GAGNÉ, C. L. (2000). Relation-based combinations versus propertybased combinations: A test of the CARIN theory and dual-process theory of conceptual combination. Journal of Memory \& Language, 42, 365-389.

GAGNÉ, C. L. (2001). Relation and lexical priming during the interpretation of noun-noun combinations. Journal of Experimental Psychology: Learning, Memory, and Cognition, 27, 236-254.

GAGNÉ, C. L. (in press). Metaphoric interpretation of comparison-based combinations. Metaphor \& Symbol.

GAGNÉ, C. L., \& MurPhy, G. L. (1996). Influence of discourse context on feature availability in conceptual combination.Discourse Processes, 22, 79-101.
Gagné, C. L., \& Shoben, E. J. (1997). The influence of thematic relations on the comprehension of modifier-noun combinations. Journal of Experimental Psychology: Learning, Memory, \& Cognition, 23, 7187.

Gelman, S., \& Markman, E. (1985). Implicit contrast in adjectives vs. nouns: Implications for word learning. Journal of Child Language, 12, 125-143.

GerRig, R. J., \& Bortfeld, H. (1999). Sense creations in and out of discourse contexts. Journal of Memory \& Language, 41, 457-468.

Gerrig, R. J., \& Murphy, G. L. (1992). Contextual influences on the comprehension of complex concepts. Language \& Cognitive Processes, 7, 205-230.

Kaschak, M. P., \& Glenberg, A. M. (2000). The role of affordances and grammatical constructions in sentence comprehension. Journal of Memory \& Language, 43, 508-529.

LEVI, J. (1978). The syntax and semantics of complex nominals. New York: Academic Press.

Meyer, D. E., \& Schvaneveldt, R. W. (1971). Facilitation in recognizing pairs of words: Evidence of dependence between retrieval operations. Journal of Experimental Psychology, 90, 227-234.

Meyer, D. E., Schvaneveldt, R. W., \& Ruddy, M. G. (1974). Functions of graphemic and phonemic codes in visual word-recognition. Memory \& Cognition, 2, 309-321.

Murphy, G. L. (1988). Comprehending complex concepts. Cognitive Science, 12, 529-562.

Murphy, G. L. (1990). Noun phrase interpretation and conceptual combination. Journal of Memory \& Language, 29, 259-288.

Neely, J. H. (1977). Semantic priming and retrieval from lexical memory: Role of inhibitionless spreading activation and limited capacity attention. Journal of Experimental Psychology: General, 106, 226254.

Onifer, W., \& Swinney, D. (1981). Accessing lexical ambiguities during sentence comprehension: Effects of frequency of meaning and contextual bias. Memory \& Cognition, 9, 225-236.

Shoben, E. J., \& GAgné, C. L. (1997). Thematic relations and the creation of combined concepts. In T. B. Ward, S. M. Smith, \& J. Vaid (Eds.); Creative thought: An investigation of conceptual structures and processes (pp. 31-50). Washington, DC: American Psychological Association.

Smith, E. E., \& Osherson, D. N. (1984). Conceptual combination with prototype concepts. Cognitive Science, 8, 337-361.

Smith, E. E., Osherson, D. N., Rips, L. J., \& Keane, M. (1988). Combining prototypes: A modification model. Cognitive Science, 12, 485527.

WISNIEWSKI, E. J. (1996). Construal and similarity in conceptual combination. Journal of Memory \& Language, 35, 434-453. 
APPENDIX

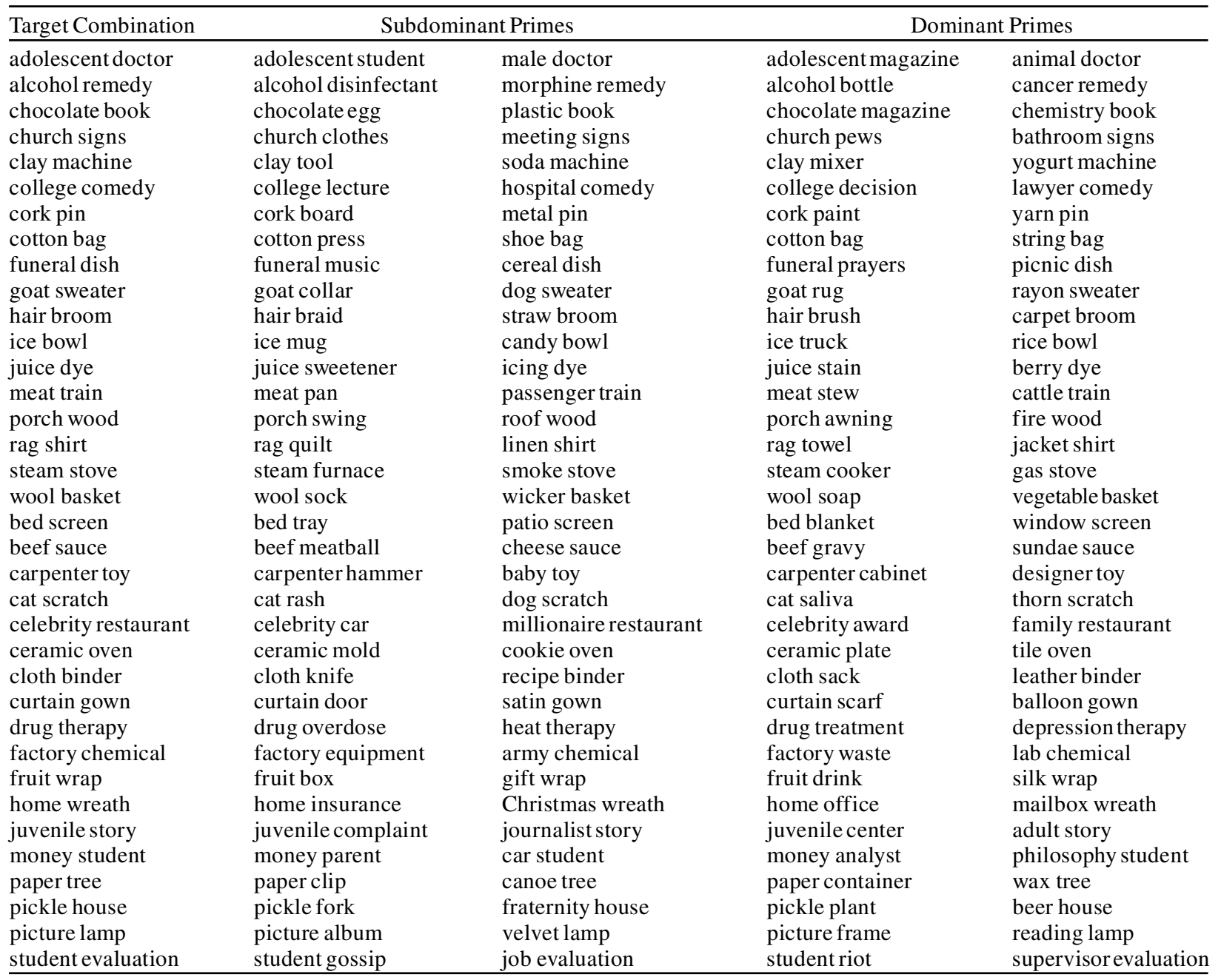

(Manuscript received September 14, 2000;

revision accepted for publication January 14, 2002.) 